BUHEP-97-24

\title{
Towards an Effective Particle-String Resolution of the Cosmological Constant Problem
}

\author{
Raman Sundrum \\ Department of Physics \\ Boston University \\ Boston, MA 02215, USA \\ email: sundrum@budoe.bu.edu
}

\begin{abstract}
The Cosmological Constant Problem is re-examined from an effective field theory perspective. While the connection between gravity and particle physics has not been experimentally probed in the quantum regime, it is severely constrained by the successes of Standard Model quantum field theory at short distances, and classical General Relativity at large distances. At first sight, it appears that combining particle physics and gravity inevitably leads to an effective field theory below the weak scale which suffers from large radiative corrections to the cosmological constant. Consequently, this parameter must be very finely tuned to lie within the experimental bounds. An analog of just this type of predicament, and its resolution, are described in some detail using only familiar quantum field theory. The loop-hole abstracted from the analogy is the possibility of graviton "compositeness" at a scale less than $10^{-2} \mathrm{eV}$, which cuts off the large contributions to the cosmological constant from standard model physics. Experimentally, this would show up as a dramatic breakdown of Newton's Law in upcoming sub-centimeter tests of gravity. Currently, strings are the only known example of such compositeness. It is proposed that the gravitational sector comprises strings of very low tension, which couple to a stringy "halo" surrounding each point-like standard model particle.
\end{abstract}




\section{Introduction}

A naturalness problem is like the sight of a needle standing upright on a table; it is consistent to assume a delicate balance, but one strongly suspects an invisible stabilizing force. The balance one must explain has the form of an extremely fine cancellation between large virtual contributions to an observable from physics at very different length scales. The grandest and most baffling of all the naturalness problems in fundamental physics is the Cosmological Constant Problem (CCP). It emerges upon putting together the two separately successful parts of our physical understanding: classical General Relativity and the quantum field theoretic Standard Model (SM). Ref. [1] provides a good review. The problem is so tightly constrained that one can hope that its final resolution will reveal an essentially unique and qualitatively new stabilizing mechanism.

Here is an outline of the problem. The classical theory of general relativity that has been tested at long distances can be thought of as the result of integrating out short distance quantum fluctuations from both the SM and gravitational sectors. Einstein's equations describing the leading longdistance behavior of the metric field, $g_{\mu \nu}$, are,

$$
R_{\mu \nu}-\frac{1}{2} g_{\mu \nu} R=\frac{1}{4 M_{P l}^{2}}\left[\lambda g_{\mu \nu}+T_{\mu \nu}\right]
$$

Here $R_{\mu \nu}$ and $R$ are the curvature tensor and scalar respectively, $T_{\mu \nu}$ is the classical energy-momentum tensor for the matter and radiation in the universe, Newton's constant has been written in terms of the Planck mass, $M_{P l}$, and $\lambda$ is the cosmological constant.1 $\lambda$ is very sensitive to the short distance physics which has been integrated out. The SM contributes its "vacuum energy", very roughly given by,

$$
\lambda_{S M} \sim \mathcal{O}\left(v^{4}\right),
$$

where $v$ is the scale of electroweak symmetry breaking. The other contributions are from less well-understood sources, namely short-distance quantum gravity and particle physics beyond the standard model.[2 However, as long

\footnotetext{
${ }^{1}$ This definition of the cosmological constant differs by a factor of $4 M_{P l}^{2}$ from the astrophysical convention, in order to give $\lambda$ the units of energy-density.

${ }^{2}$ My language assumes that the standard model (possibly with the exclusion of the physical Higgs degree of freedom) is just an effective theory valid below roughly $v$, and is superseded by some other theory at higher energies.
} 
as these exotic contributions do not unexpectedly finely cancel the SM contribution, we must have the following rough lower bound on the cosmological constant,

$$
|\lambda| \geq \mathcal{O}\left(v^{4}\right)
$$

On the other hand, in solutions to eq. (1), $\lambda$ contributes to the cosmological expansion rate. This permits a conservative bound to be put on $\lambda$ by using the measured expansion rate of the universe and estimates of $T_{\mu \nu}$. With high confidence, the experimental bound is given by

$$
|\lambda|<10^{-56} v^{4}
$$

Now, eqs. (3) and (4) are in wild disagreement. To lower the bound in eq. (3) sufficiently to accord with eq. (4) requires an unbelievably fine cancellation between the contributions to $\lambda$ from quantum fluctuations below and above the weak scale. This is the CCP.

The attitude taken in this paper is that some part of the preceding story is simply wrong, and the true story must eliminate the need for fine-tuning in order to obtain an acceptably small value for $\lambda$. It is frequently believed that the true account cannot be understood by conventional means. According to this view, the resolution of the CCP may not be expressible in terms of the local quanta and interactions of a relativistic quantum theory. This is not the viewpoint of the present paper; the fundamental principles of relativity, quantum mechanics and locality are central to the understanding of the CCP, and the proposed resolution does not transcend them.

However, the CCP as described above is extremely robust, based only on the co-existence of gravity and mass scales of order $v$ (and supersymmetrybreaking of at least the same magnitude). The CCP then follows by elementary power-counting. Section 2 of this paper describes the CCP in greater detail using effective field theory methodology. Effective field theory provides a clear and economical separation of the facts and principles which we have already tested experimentally, from the physics which is still beyond our reach, both in the gravitational and particle physics sectors. It is a useful language for examining assumptions which we may need to discard, as well as for evaluating new proposals for solving the CCP.

In order to solve the CCP we must change the power-counting which determines how sensitive to SM mass scales the long-distance theory of eq. (1) is. In quantum field theory, whenever the physics of a large mass scale is integrated out, the sensitivity of the low-energy effective theory is determined by power-counting for the weakly-coupled degrees of freedom at that mass 
scale, not just the degrees of freedom at the lowest energies. To use this observation in the case of the CCP, we must ensure that at particle physics energies, the gravitational degrees of freedom are profoundly different from those in eq. (1). We can loosely speak of the graviton as a "composite" of these new degrees of freedom. How can this crucial new physics be right underfoot without our having noticed, and how exactly can compositeness help with the CCP? Section 3 provides a detailed analogy of the CCP where these questions, and others, can be understood in the context of a simple toy universe. This serves as a useful warm-up because the resolution of the toy naturalness problem is based on completely familiar physics.

Finally, in section 4, a possible new mechanism for stabilizing an acceptably small cosmological constant is put forward. Below the weak scale, it consists of a gravitational sector made of extremely low-tension strings, with string-scale less than $10^{-2} \mathrm{eV}$, interacting with the stringy "halos" carried by SM particles. The low string tension cuts off the virtual contributions to $\lambda$ so that it naturally satisfies eq. (4). At very large distances only the massless string mode, namely the graviton, is relevant, and the dynamics reduces to general relativity. In accelerator experiments, the macroscopic string halo carried by particles is unobserved because the stringy gravitational physics is too weakly coupled to compete with point-like SM interactions. The detailed structure of such an effective particle-string theory has not yet been worked out, but I discuss its necessary properties as well as possible directions towards its construction. If this scenario is correct there will be a striking experimental signature: Newton's Law will completely break down when gravity is tested at sub-centimeter distances!

This proposal may appear heretical from the view of traditional field theory and string theory. However, recent developments in string theory offer some encouragement. There is evidence that strings can co-exist with objects of different dimensionality, D-branes, including 0-branes which are point-like states. For a review see ref. [2]. There have already been several calculations of the scattering of D-branes with strings and with each other, which reveal a stringy halo about the D-branes [3] [ [⿴囗十 . These results may be useful for constructing effective particle-string theories. Refs. [5] are some initial forays in this direction.

However, I wish to point out an important difference between the goal of the present work and the goal of most of the string literature. The recent string theory upheaval is part of a very ambitious program aimed at a nonperturbative understanding of fundamental interactions at at the highest energies. On the other hand, the CCP is a puzzle whose answer lies at 
present-day energies, but is presumably hidden from view because of the weakness of the gravitational force. The purpose here is to develop an effective theory which has an ultraviolet cutoff given by the weak scale, and whose parameters can naturally be fit to experiment. The effective theory is permitted to break down above the weak scale, and be replaced by a more fundamental description there.

Sections 2, 3 and 4 may be read in any order depending on the background and interests of the reader. Section 5 provides the conclusions.

I make use of rough estimates in several places. It is customary when power-counting to keep track of factors of $4 \pi$ that arise from the dimensionality of space-time. In this paper, I will consider all such factors to be order one since the Cosmological Constant Problem involves such large numbers that, by comparsion, $4 \pi$ factors are unremarkable. When estimating Feynman diagrams, dimensional regularization is implicit for simplicity. This will not remove any important physics (for example it does not eliminate the CCP) because the important mass scales will be explict and will not need to be represented by a dimensionful cutoff.

\section{The Problem in Context}

\subsection{The standard effective theory of particles and gravity}

The most straightforward way to put together the SM and general relativity is to write the lagrangian

$$
\mathcal{L}_{e f f}(v)=\sqrt{-g}\left\{\lambda_{0}+2 M_{P l}^{2} R+\mathcal{L}_{S M}+\ldots\right\},
$$

where $g_{\mu \nu}$ appears in $\mathcal{L}_{S M}$, minimally coupled to maintain general covariance. [I In order to compute quantum mechanical fluctuations of the metric around a Minkowski space vacuum we note that,

$$
g_{\mu \nu}=\eta_{\mu \nu}+\frac{h_{\mu \nu}}{M_{P l}}
$$

where $\eta_{\mu \nu}$ is the Minkowski metric, and $h_{\mu \nu}$ is the canonically normalized spin-2 graviton field. For most regions of spacetime, this weak field expansion about a Minkowski metric is justified. The broad perspective of general relativity adopted in this paper, as a phenomenological theory of gravity, is

\footnotetext{
${ }^{3}$ To be more precise, for fermions we must work in terms of the vierbein, but this detail is inessential in this paper.
} 
detailed in refs. [6] [7] [8]. It is based on the generally observed principles of relativity and quantum mechanical unitarity.

As is well-known, the inclusion of gravity renders the lagrangian nonrenormalizeable by elementary power-counting. This means that the resultant theory cannot be a fundamental description of nature at all energies (at least perturbatively). However, the lagrangian is a sensible basis for a quantum theory effective at energies far below the Planck scale, $M_{P l}$. Recall how this works in a general non-renormalizeable theory. Technically a nonrenormalizeable theory requires an infinite number of counterterms, which at first sight appears disasterous. The situation greatly improves if we restrict ourselves to physical processes at energies, $E$, far below the (smallest) mass scale suppressing the non-renormalizeable interactions, $M$. This allows us to work to any fixed order in the small parameter $E / M$, say $\mathcal{O}\left(\left(\frac{E}{M}\right)^{n}\right)$. To this order only the finite number of interactions and counterterms of dimension less than or equal to $n+4$ are relevant. While this statement is rather obvious at tree-level, non-trivially it survives loops and renormalization. For $E \ll M$ we thereby obtain a well-defined and predictive effective field theory. The effective theory must give way to a more fundamental description at some scale below $M$, or perhaps be sensible but strongly-coupled above $M$. The best known example of a non-renormalizeable effective field theory is the chiral lagrangian description of pions, treated as Nambu-Goldstone bosons of chiral symmetry breaking. For a review see ref. [9]. The typical scale appearing in the non-renormalizeable interactions is the hadronic scale, $M \sim 1$ $\mathrm{GeV}$. The effective field theory is therefore sensible and weakly-coupled for $E \ll 1 \mathrm{GeV}$. For $E>1 \mathrm{GeV}$, the effective theory fails completely and must be replaced by the more fundamental QCD description.

In the case at hand, the scale suppressing the non-renormalizeable interactions is $M_{P l}$. Therefore the theory given by eq. (5) makes sense at energies $E \ll M_{P l}[8]$. In fact let us take the ultraviolet cutoff of our effective theory to be the weak scale, as denoted by the $v$ appearing on the left-hand side of eq. (5). This allows us to remain agnostic about the nature of physics beyond the weak scale. The ellipsis in eq. (5) can contain higher-dimension gauge and coordinate invariant interactions, whose effects are small at energies far below the weak scale.

As far as accelerator experiments are concerned, eq. (5), provides a very economical summary of what has been actually observed. They overwhelm-

\footnotetext{
${ }^{4}$ More precisely, they are irrelevant in that their dominant effects can be absorbed into finite renormalizations of the lower dimension interactions.
} 
ingly confirm a relativistic quantum field theory given by the SM for energies below the weak scale, with gravitational forces being negligible. Thus the "laboratory tested" part of eqs. $(5,6)$ is given by the formal limit,

$$
\mathcal{L}_{e f f}^{l a b}(v) \rightarrow \mathcal{L}_{S M}, \quad g_{\mu \nu} \rightarrow \eta_{\mu \nu}, \quad \text { as } 1 / M_{P l} \rightarrow 0 .
$$

At macroscopic distances, with large amounts of matter and radiation, the SM forces are effectively neutralized and gravity dominates. Because of the large distances, masses and numbers of quanta, the classical approximation is justified. Conceptually, one arrives at a classical effective theory for this regime by integrating out all quantum fluctuations from eq. (5). The result must have the form of classical general relativity, eq. (1). This is because eq. (1) is the most general form consistent with the general covariance of our starting point, eq. (5), up to terms involving higher-dimension metric invariants which are irrelevant at macroscopic distances.

It is important to note that only the classical macroscopic effective theory, rather than the full effective quantum field theory of eq. (5), has been tested gravitationally. This is in contrast to the SM sector, where the full quantum field theoretic implications of eq. (5) have been tested. Therefore we must bear in mind that while eq. (5) is in accord with all gravitational tests since it reduces to eq. (1), the "bare" parameter $\lambda_{0}$ allowing us to fit the experimental bound of eq. (4), eq. (5) may not be unique in this respect.

It is somewhat of a nuisance that eq. (1) combines two steps in its derivation, the integrating out of microscopic physics and the classical limit for large numbers of quanta. It is useful to separate the two issues by considering a long distance effective theory in a simplified setting, involving just a few SM particles, but treated fully quantum field theoretically. I will develop such an effective theory in the next subsection. It will provide a useful point of contact when we discuss the analogy in Section 3.

\subsection{A macroscopic quantum effective lagrangian with gravity}

Consider a few stable massive spin-0 particles, $H$, with relative momenta only of order $\mu \ll v$, interacting with soft gravitons with energies of order $\mu$. The length scale, $1 / \mu$ will act as our short-distance cutoff. We can take $1 / \mu \sim 1 \mathrm{~mm}$, which is less than the shortest range over which gravity has been tested. One can imagine $H$ to be a ground state hydrogen atom say, whose compositeness cannot be resolved by the long wavelength gravitons. 
Alternatively we can take $H$ to be just a proxy for more fundamental particles like an electron, neglecting the complications of spin and charge. For simplicity I will also neglect the other soft massless particles, photons and neutrinos. We therefore have an isolated sector which should be described by an effective lagrangian containing only the $H$ and $g_{\mu \nu}$ fields. This type of theory is entirely analogous to the heavy particle effective theories used in studying the strong interactions, where soft pions interact with a massive hadron, or gluons interact with a heavy quark. This is reviewed in ref. [9]. I will simply take over the methodology to the case at hand.

The first observation is that since $m_{H} \gg \mu$, the 4-velocity of $H, v_{\mu}$, is approximately conserved in collisions with soft gravitons, to within $\mathcal{O}\left(\mu / m_{H}\right)$. Thus the $H$ momenta have the form,

$$
p_{\mu}=m_{H} v_{\mu}+k_{\mu} ; \quad k_{\mu} \sim \mathcal{O}(\mu) .
$$

For the simple case considered here, $v_{\mu}$ is common to all the $H$ particles involved, since their relative momenta were assumed to be of order $\mu$. We perform a field redefinition of the scalar field to remove the large fixed component of the momentum, $m_{H} v$,

$$
H_{v}(x) \equiv \sqrt{2 m_{H}} e^{-i m_{H} v_{\mu} x^{\mu}} H(x) .
$$

The $H_{v}$ field thereby has residual momentum $k_{\mu} \sim \mathcal{O}(\mu)$, just like the gravitons. Because $v_{\mu}$ is a Minkowski space vector, not a generally covariant vector, it is important to note that the generally covariant derivative for $H_{v}$ is $i m_{H} v_{\mu}+\partial_{\mu}$, rather than just $\partial_{\mu}$ for $H$.

The general form of the $\mu$-scale effective lagrangian in this sector is,

$$
\begin{aligned}
\mathcal{L}_{e f f}(\mu) & =\sqrt{-g}\left\{\lambda+2 M_{P l}^{2} R+\frac{1}{2 m_{H}} g^{\mu \nu}\left(-i m_{H} v_{\mu}+\partial_{\mu}\right) \bar{H}_{v}\left(i m_{H} v_{\nu}+\partial_{\nu}\right) H_{v}\right. \\
& \left.-\frac{m_{H}}{2} \bar{H}_{v} H_{v}+\ldots\right\} .
\end{aligned}
$$

The effective lagrangian is manifestly generally coordinate invariant, a symmetry of the starting point, eq. (5). The ellipsis contains higher dimension operators constructed from $g_{\mu \nu}$ and $H_{v}$, including local $H$ self-interactions, whose effects are small at large distances (but can be systematically included). If $\lambda=0$, we can expand the quantum theory about a Minkowski vacuum, $g_{\mu \nu}=\eta_{\mu \nu}, H_{v}=0$. In the frame of the $H$ particles, given by $v=(1, \overrightarrow{0})$, eq. (10) then becomes,

$$
\mathcal{L}_{e f f}(\mu)=\sqrt{-g}\left\{2 M_{P l}^{2} R+\bar{H}_{v}\left(i \partial_{0}+\frac{\partial^{2}}{2 m_{H}}\right) H_{v}-\frac{m_{H}}{2 M_{P l}} h^{00} \bar{H}_{v} H_{v}\right.
$$




$$
\left.+\mathcal{O}\left(\frac{\mu}{M_{P l}}\right)+\ldots\right\}
$$

describing non-relativistic $H_{v}$ particles coupled to gravity, predominantly through their gravitational "charge", $m_{H} / M_{P l}$. After gauge-fixing the gravitational fields (see for example ref. [7]]), we can integrate out 1-graviton exchange which dominates the $H_{v}$ interactions, to obtain a non-local Newtonian potential interaction between $H_{v}$ particles. If $\lambda \neq 0$, the field theory must be expanded about an "expanding universe" metric rather than Minkowski space.

Since eq. (10) describes a quantum field theory, we may ask if $\lambda$ can naturally be as small as eq. (4) under quantum corrections within this effective theory. The answer is yes! Conceptually, all the fields in eq. (10) have their momenta cut off at $\mu$, because of the field redefinition, eq. (9). Thus although by power-counting we estimate that $\lambda$ should get radiative corrections of order four powers of the cutoff, this is just $\mu^{4}$, which for $1 / \mu \sim 1 \mathrm{~mm}$, is well within the experimental bound, eq. (4). In fact if we adopt dimensional regularization, the $H_{v}$-loop corrections to $\lambda$ vanish.

The effective field theory described above reproduces some familiar phenomena of classical general relativity, such as the Newtonian force between non-relativistic masses, and gravitational radiation. On the other hand the effective theory is fully quantum mechanical and unitary in its domain of validity, and predicts inherently quantum corrections to the classical approximation. (An example of such corrections is described in ref. [8].) Yet, it has a naturally small cosmological constant. For these reasons it is a useful conceptual link between microscopic physics and classical general relativity.

\subsection{The Cosmological Constant Problem}

The $\mu$-scale effective theory given by eq. (10) and the classical effective theory of eq. (1) both share the same cosmological constant, $\lambda$. I will focus on eq. (10) since it is a straightforward quantum effective field theory, though analogous statements follow for classical general relativity, eq. (1). If we do not look beyond the effective theory of eq. (10), $\lambda$ can naturally satisfy the experimental bound, eq. (4), as pointed out above. However, our present point of view is that $\lambda$ is determined by matching the effective theory of eq. (10) with the more fundamental theory of eq. (5), or conceptually, by integrating out the physics below $v$. If one fixes a particular regularization and renormalization scheme, say dimensional regularization with minimal subtraction, one can actually perform the matching computations. Here we 
only require the results of simple power-counting, which shows that $M_{P l}$ is negligibly renormalized in matching at $\mu$, while by contrast, $\lambda$ is quartically sensitive to the mass scales of the SM, so that,

$$
\lambda=\lambda_{0}+\mathcal{O}\left(v^{4}\right)
$$

We have no way of understanding why the physics above the weak scale, which determines $\lambda_{0}$, should so precisely cancel against the $\mathcal{O}\left(v^{4}\right) \mathrm{SM}$ contributions, in order for eq. (4) to hold.

Thus I conclude, although eq. (5) reduces to the SM at short distances, (eq. (7)), reproducing all accelerator experiments, and though it reduces to eqs. (1) and (10) at macroscopic distances, thereby accomodating all gravitational measurements, it is not the correct effective theory below the weak scale because it involves a fantastic and inexplicable fine-tuning of $\lambda$. We must therefore see what room we have for changing the weak scale theory without destroying its highly non-trivial theoretical consistency and agreement with experiment.

What seems highly significant to me is this. The cosmological constant is usefully thought of as a non-derivative graviton self-coupling (which destabilizes Minkowski spacetime). Quantum corrections to $\lambda$ come from loops of massive SM states, coupled to external graviton lines at essentially zero momentum. Therefore necessarily, these massive SM states are far off-shell. On the other hand, experimentally we have only tested the gravitational couplings of SM states which are very nearly on-shell. . For example, the $H$ particles of the previous subsection are very nearly on-shell in the domain of validity of the $\mu$-scale effective theory. It follows that all the large quantum corrections to $\lambda$ from the weak-scale theory of eq. (5) come from a tremendous theoretical extrapolation to the regime where gravitons couple to SM particles which are far off-shell. We can hold out some hope that the CCP can be avoided by a different weak scale effective theory, which however still reduces to eqs. (1) and (10) in the domain of on-shell SM matter coupled to soft gravitons.

\subsection{Constraints on alternative weak-scale theories}

In thinking about alternative effective theories, it is crucial to observe two powerful fundamental principles, at least as far as physics below the weak

\footnotetext{
${ }^{5}$ By contrast note that accelerator experiments have very successfully probed highly virtual, purely SM effects, in the form of running couplings and precision electroweak tests.
} 
scale is concerned. First, to quite large distances, spacetime appears as a Minkowski continuum. It also appears to be true down to distances of order $1 / v$, since the highly successful SM loop computations depend sensitively on this assumption. Secondly, nature is quantum mechanical, at least up to weak scale energies. Furthermore, it is difficult to perturb the quantum principle withut leading to physical absurdities. Therefore it would appear that we cannot seriously doubt the principles of (local) special relativity and quantum mechanics in the gravitational sector below the weak scale. These two principles impose very severe constraints on model-building. Taken with the experimental success of general relativity at large distances they necessarily imply the existence of a massless spin-two particle, the graviton, which must underlie any effective theory of gravity. Furthermore, this effective theory must obey the gauge symmetry of general coordinate invariance [10]. This is similar to the case of light spin-one particles, where a gauge symmetry is needed to decouple unphysical degrees of freedom, but in the case of spin-two the gauge symmetry is unique!

Now, if we restrict ourselves to the minimal particle content, namely the SM particles and the graviton, the form of the effective theory is given by eq. (5), this being the most general invariant form that reduces to the SM when $1 / M_{P l} \rightarrow 0$, and containing the kinetic term for the graviton field. But eq. (5) is just the effective theory we are trying to avoid. Thus we conclude that new particles associated with gravity must be present. They must be very light indeed in order to remain in the effective theory down to the very low energies necessary to protect the cosmological constant, as has recently been emphasized in ref. [11].

Unfortunately, all proposals to couple extra particles to eq. (5) have failed to cure the naturalness problem. Generic addition of extra light particles does not evade the simple power-counting which says that the cosmological constant is quartically sensitive to the highest mass scales in the theory. Supersymmetrizing eq. (5) does in fact stabilize a suitably small $\lambda$. However this requires supersymmetry to be unbroken in the SM sector to very high precision, in order to suitably reduce the $\mathcal{O}\left(v^{4}\right)$ contribution in eq. (12). Experimentally however, we know that supersymmetry is badly broken in this sector. Other than supersymmetry the only other special symmetry

\footnotetext{
${ }^{6}$ There have been suggestions that general coordinate invariance can be replaced by restricted invariance under coordinate transformations with unit Jacobian. However, both classically and quantum mechanically this is precisely equivalent to a generally invariant theory with an arbitrary (but not naturally small) cosmological constant. See ref. [1] for a brief review, plus references.
} 
that can control the cosmological constant is conformal symmetry. This is also badly broken in nature, but there have been several attempts to make $\lambda$ a dynamical field that relaxes to zero as a consequence of the conformal anomaly, similarly to the way an axion can relax the strong interactions to a CP-conserving vacuum in the presence of a $\theta$ angle, as a consequence of the axial anomaly. For the CCP all such attempts have failed for the general reason described in ref. [1]. To summarize, while we can always weakly couple eq. (5) to new light particles, there is no reason for these to significantly reduce the $\mathcal{O}\left(v^{4}\right)$ SM loop contributions to $\lambda$.

The seemingly impossible predicament posed by the CCP has given rise to proposals which play by different rules from those we have adopted. They cannot be evaluated within any local effective theory and are difficult to test experimentally. It is possible that one of these proposals is nevertheless true. Perhaps the best-known is Coleman's wormhole proposal [12] [1]. Here, wormhole physics, just below the Planck scale, gives rise to peculiar nonlocal effects (from the viewpoint of our macroscopic spacetime), whereby the fundamental "constants" of nature become dynamical variables, but without any local spacetime variations. The relevant path integral is infinitely peaked at values of these constants such that the bottom-line cosmological constant vanishes, $\lambda=0$.

The present paper describes a deliberately restricted search for a resolution of the CCP which can be described by a natural effective theory, expressed in terms of local degrees of freedom. This is the time-honored approach taken towards other naturalness problems such as the the Strong $\mathrm{CP}$ problem or the Higgs naturalness problem. However, the arguments of this section seem to suggest that we are at an impasse. There may be a way out though, as suggested by the following parable.

\section{An Analogy}

In this section I describe a naturalness problem, analogous to the CCP, which occurs within a toy model universe. This toy problem has the advantage of involving only the familiar quantum field theory of particles with spins less than or equal to one. Nevertheless, the resolution sheds light on how the CCP might be resolved. The model consists of two sectors, a toy "Standard Model" (TSM) accounting for short-distance "laboratory" physics, and a toy "gravity" (TG) only noticeable at very large distances.

The TSM is simply the quantum electrodynamics of eight identical fla- 
vors of charged fermions, $\psi_{a}, a=1, \ldots, 8$. I will cut off the electromagnetic interactions at larger than laboratory distances by giving the photon a very small mass.] Recall that for an abelian gauge field, a mass term is both renormalizable and naturally small (only receiving logarithmic quantum corrections). The renormalizeable and natural TSM theory is then given by

$$
\mathcal{L}_{T S M}=\bar{\psi}_{a}(i \not D-m) \psi_{a}-\frac{1}{4} F_{\mu \nu}^{2}-\frac{m_{A}^{2}}{2} A_{\mu}^{2},
$$

with $m_{A} \ll m, \alpha_{e m}(m) \ll 1$. We will consider the TSM to have been tested at lab momenta, very roughly of order $m$ (where the photon mass is negligible), and to a precision given by $\epsilon \ll \alpha_{e m} \ll 1$. For example, our momentum resolution is of order $\epsilon m$, and we are insensitive to $n$-loop QED effects for $n$ such that $\alpha_{e m}^{n}<\epsilon$. Nevertheless I will consider $\epsilon$ to be small enough that eq. (13) has been non-trivially tested as a quantum field theory.

On the other hand, TG corresponds to the observation of a very weak classical scalar Yukawa force, $y^{2} e^{-m_{\phi} r} / r^{2}$, between non-relativistic $\psi$ particles§ over very large distances and times,

$$
r, t \gg \frac{1}{m_{\phi}} \gg \frac{1}{m_{A}}
$$

Notice this implies that the exponential suppression is always turned on in the Yukawa force, but clearly it is still the dominant force at very large distances. The TG force is too weak to be observed at short distances in the lab, against the background of electromagnetism, but is seen outside the photon range. To be concrete let us take,

$$
y^{2} \sim \epsilon
$$

The mass scale $m_{\phi}$ is extremely small,

$$
m_{\phi} \ll m e^{-1 / \epsilon} .
$$

At the purely classical level this is acceptable, as is a very small or zero cosmological constant in classical general relativity.

\footnotetext{
${ }^{7}$ Of course, in the real world the electromagnetic force is negligible on large distance scales because of the neutrality of massive gravitating objects, like planets and stars. The toy photon mass makes for a simpler story.

${ }^{8}$ Unlike the real world, in the toy universe the photon does not "gravitate".
} 
The minimal relativistic quantum field theory incorporating both the TSM and TG necessarily associates a scalar field, $\phi$, with the Yukawa force,

$$
\mathcal{L}=\bar{\psi}_{a}(i \not D-m+y \phi) \psi_{a}-\frac{1}{4} F_{\mu \nu}^{2}-\frac{m_{A}^{2}}{2} A_{\mu}^{2}+\frac{1}{2}\left(\partial_{\mu} \phi\right)^{2}-\frac{m_{\phi}^{2}}{2} \phi^{2}-g_{\phi} \phi^{4},
$$

where the scalar coupling $g_{\phi}$ is included for renormalizability, though it is too small to observe and plays no further role. Eq. (17) is the analog of eq. (5). Like eq. (5), it suffers from a naturalness problem. Here, the problem is why the scalar mass, $m_{\phi}$, is so small, despite much larger quantum corrections coming from TSM $\psi$ loops. Standard power-counting and eqs. $(15,16)$ give,

$$
\delta m_{\phi}^{2} \sim y^{2} m^{2} \gg m_{\phi}^{2}
$$

Physicists of the toy universe may note that a small scalar mass is stabilized by supersymmetry. But the fact that no $\psi$ superpartners have been observed for energies well above $m$ means that supersymmetry is badly broken, and eq. (18) still holds. This is closely analogous to the situation with the cosmological constant and supersymmetry in the real world.

Other than supersymmetry there is no mechanism by which a weakly coupled fundamental scalar can naturally avoid corrections like eq. (18). One might think that the spin-0 particle could be fundamental and light if it is a Nambu-Goldstone boson of a spontaneously broken symmetry, but this possibility can be ruled out as well. Even though fundamental NambuGoldstone bosons are naturally massless, their "decay constants" are naturally of order the highest scale in the theory, in the present case, $f_{\pi} \sim m$. The fact that the spin-0 particle has non-derivative couplings to the $\psi$ 's means that the spontaneously broken symmetry must also be explicitly broken. The same explicit breaking which gives rise to a Yukawa coupling, $y$, naturally gives rise to a pseudo-Nambu-Goldstone boson mass-squared of order $y f_{\pi}^{2} \sim y m^{2}$, which is incompatible with eq. (16).

The only remaining means of obtaining a naturally light spin-0 particle is to make it a composite, like a hadron, with a very low compositeness scale. Even this approach offers no comfort at first sight. The basic reason is that the $\phi$ self-energy estimate due to TSM loops, yielding eq. (18), is performed at essentially zero external momentum, and so is completely insensitive to whether $\phi$ is composite or fundamental. Thus in any model where $\phi$ has a low enough compositeness scale to naturally satisfy eq. (16), it will be impossible to arrange for a Yukawa coupling as large as in eq. (15). I will illustrate this with a specific example. Suppose we try to make $\phi$ a scalar 
glueball of a Yang-Mills sector, with a confinement scale $\Lambda_{Y M} \ll m e^{-1 / \epsilon}$, which sets the glueball mass. To obtain a Yukawa coupling to the $\psi$ 's we can use a higher dimension interaction,

$$
\delta \mathcal{L}=h \frac{\bar{\psi} \psi \operatorname{tr} G_{\mu \nu} G^{\mu \nu}}{m^{3}}
$$

where $h$ is a dimensionless coupling, and $\operatorname{tr} G_{\mu \nu} G^{\mu \nu}$ is the Yang-Mills operator that interpolates the glueball, $\phi$,

$$
<0\left|\operatorname{tr} G_{\mu \nu} G^{\mu \nu}\right| \phi>\sim \Lambda_{Y M}^{3} .
$$

Recall that a non-renormalizable interaction such as eq. (19) is acceptable within effective field theory. Taking the effective theory cutoff to be of order $m$, the energy scale probed in the lab, the theory remains weakly coupled at the cutoff provided $h \ll 1$. (In fact we must have $h<\epsilon$ in order for the $\psi$-gluon interactions to have not been directly seen in the lab.) Therefore we arrive at the unsatisfactory result,

$$
y \sim h \frac{\Lambda_{Y M}^{3}}{m^{3}} \ll e^{-3 / \epsilon},
$$

in contradiction to eq. (15).

I hope to have convinced the reader that, like the CCP, this toy naturalness problem seems to leave no room for manoeuvre. However, this is a false impression.

Fortunately, compositeness does allow the resolution of the naturalness problem. In order to invalidate the reasoning behind the large quantum corrections to $m_{\phi}$ from the TSM, we must not only take $\phi$ to be a composite light hadron, but we must also consider the massive particles it interacts with in TG to be heavy hadrons containing the TSM $\psi$ particles as heavy quarks! The specific resolution I have in mind is given by,

$$
\begin{aligned}
\mathcal{L} & =\bar{\psi}(i \not D-m) \psi+\bar{u}\left(i \not D-m_{u}\right) u+\bar{d}\left(i \not D-m_{d}\right) d \\
& -\frac{1}{4} F_{\mu \nu}^{2}-\frac{m_{A}^{2}}{2} A_{\mu}^{2}-\frac{1}{4} \operatorname{tr} G_{\mu \nu}^{2}+\frac{\theta \alpha_{s}}{8 \pi} \operatorname{tr} \tilde{G}_{\mu \nu} G^{\mu \nu}
\end{aligned}
$$

where I have introduced an $S U(3)_{Q C D}$ gauge theory, under which the $u$ and $d$ quarks are triplets, and the eight $\psi_{a}$ TSM fields form an $S U(3)_{Q C D}$ adjoint representation. Only the $\psi$ 's are electrically charged however.

The $\phi$ is a particular combination of the three QCD pions, $\vec{\pi}$, made out of $u$ and $d$ quarks, while the heavy fermion it interacts with hadronically in TG, 
$\Psi$, is a composite of the adjoint $\psi$ quark and glue. The light quark masses $m_{u, d} \ll \Lambda_{Q C D}$ are needed to produce non-derivative Yukawa couplings of $\phi$ to $\Psi$, and to generate a small $m_{\phi}$. The Yukawa coupling we need, $\phi \bar{\Psi} \Psi$, breaks isospin symmetry under which the pions form a triplet, whereas $\Psi$ is a singlet. The requisite isospin breaking is arranged by taking $m_{u} \neq m_{d}$. Another technicality is that QCD is normally a parity-conserving theory, so a single pseudo-scalar pion cannot couple to the scalar $\bar{\Psi} \Psi$ as required. I have therefore added an order one CP-violating $\theta$-term. Pf The resulting Yukawa coupling is then of order a small power of $m_{u, d} / \Lambda_{Q C D}$, while

$$
m_{\phi}^{2} \sim\left(m_{u}+m_{d}\right) \Lambda_{Q C D} .
$$

By taking $\Lambda_{Q C D} \sim m e^{-1 / \epsilon}$, we can consistently choose $m_{u, d} \ll \Lambda_{Q C D}$ so that $y \sim \epsilon$ and $m_{\phi} \ll m e^{-1 / \epsilon}$, as desired!

There are three issues we would like to understand better: (i) Why is the composite QCD structure not already observed in the long distance TG sector? (ii) Why is the composite structure not visible in the lab? (iii) How does the composite structure cure the $\phi$ mass of extreme sensitivity to the TSM mass scale, $m$ ? Some of the discussion will be similar to that of ref. [13], where a model with very small $\Lambda_{Q C D}$ was also considered.

(i) The interactions of very low-energy pions with slow heavy $\Psi$ hadrons can be described using a Heavy Hadron Effective Lagrangian (reviewed in ref. [9]),

$$
\mathcal{L}_{e f f}=\bar{\Psi}\left(i \partial_{0}+\frac{\partial^{2}}{2 m}+y \phi\right) \Psi+\frac{1}{2}\left(\partial_{\mu} \phi\right)^{2}-\frac{m_{\phi}^{2}}{2} \phi^{2}+\ldots
$$

Recall that $\phi$ is some linear combination of the $\vec{\pi}$ fields depending on $m_{u, d}$ and $\theta$. The ellipsis contains terms whose effects on $\Psi$ are negligible at the very low momentum transfers, $p \ll m_{\phi} \ll \Lambda_{Q C D}$, corresponding to eq. (14). These include the higher dimension couplings (suppressed by powers of $\Lambda_{Q C D}$ ) of $\phi$ to itself and to the $\Psi$, and all couplings involving $\vec{\pi}$ fields other than $\phi$. Eq. (24) is just the analog of eq. (11). Integrating out $\phi$ exchange, which dominates the $\Psi$ interactions at long distance, yields the simple Yukawa potential. The next lightest state above the $\phi$ that can be exchanged between $\Psi$ 's is a two-pion state. But in the regime of eq. (14),

\footnotetext{
${ }^{9}$ In fact, even for $\theta \neq 0$, the Yukawa coupling is not generated at first order in $m_{u, d}$ because of a vacuum re-alignment induced by $\theta$. However, the Yukawa coupling is generated at higher order in $m_{u, d}$.
} 
even the two-pion exchanges are exponentially suppressed relative to single$\phi$ exchange.

As far as eq. (24) is concerned the scalar mass is naturally small because of the very low cutoff on the effective theory. Compositeness effects are invisible because the compositeness scale is too high compared with the (virtual) $\phi$ momenta corresponding to eq. (14). We see that the firstconjectured form of the TG field theory is wrong. $\phi$ does not interact with the $\psi_{a}$ quarks, but rather with the hadronic "brown muck" of the heavy $\Psi$ hadron.

One might worry that there can be excited $\Psi$ composites, which have different Yukawa couplings $y$, but over the time scales of eq. (14) such states would decay to the lowest stable $\Psi$ state. A minor technical dynamical assumption that must be made (but which fortunately has no analog in the real (CP) is that any exotic composites of $\psi$ and two or more light quarks are heavy enough to decay to $\Psi$ via pion emission, so that their possibly different Yukawa couplings are not seen.

(ii) Typical lab momenta are of order $m$, where the running QCD coupling is weak. In the limit where it vanishes, the TSM sector completely decouples from the QCD sector. We can work out the actual value of the coupling renormalized at the laboratory momentum resolution, $\alpha_{s}(\mathrm{\epsilon m})$, using the one-loop QCD $\beta$-function and the fact that we have already chosen $\Lambda_{Q C D} \sim m e^{-1 / \epsilon}$. The result is,

$$
\alpha_{s}(\epsilon m) \sim \epsilon \ll \alpha_{e m}
$$

Therefore QCD-induced $\psi$ momentum transfers larger than $\epsilon m$ have amplitudes suppressed by $\epsilon$, so they are too small to be seen against TSM interactions. On the other hand, amplitudes where the QCD-induced momentum transfers are less than $\epsilon m$ remain unsuppressed, corresponding to soft radiation of light hadrons and excitation of the $\Psi$ resonances. But such momentum transfers are smaller than our momentum resolution. The QCD sector is therefore invisible in the lab! Note, it is only the $\psi$ particles which feel the electromagnetic force and determine the outcome of lab experiments.

In the absence of the electromagnetic interaction the $\psi$ 's would also form heavy quarkonium bound states, but with electromagnetism the QCD interactions will only negligibly perturb the electromagnetically bound states.

(iii) We now see that the unnaturally large quantum corrections in eq. (18) arose because of $\Psi$ loops, where the $\Psi$ appears far off-shell, with a Yukawa coupling to $\phi$. But this simple coupling is only valid in eq. (24), 
where the $\Psi$ is nearly on-shell. The extrapolation off-shell is completely invalid since the $\Psi$ compositeness scale is very low. The true quantum corrections to the $\phi$ mass from the TSM sector require knowledge of the full QCD dynamics. We will now correctly compute the $m_{\phi}$ sensitivity to $m$. To make the question precise let us fix some ultraviolet cutoff, $\Lambda_{0} \gg m$, relative to which we can measure masses. This could be the scale of some new physics beyond the toy standard model. We also fix $\alpha_{s}\left(\Lambda_{0}\right)$ and ask how $m_{\phi} / \Lambda_{0}$ changes as a function of $m / \Lambda_{0}$. We already have the mass formula for pseudo-Nambu-Goldstone bosons, eq. (23). We can integrate out the effects of the very heavy (adjoint) quark because of the asymptotic freedom of QCD. The dominant behavior follows from the one-loop renormalization group. The infrared renormalized quark mass parameters that appear in eq. (23) are the result of running down from $\Lambda_{0}$. To one-loop order however, this mass renormalization is independent of the heavy quark mass, $m$. Only $\Lambda_{Q C D}$ is changed at one loop because the heavy quark slows down the running of $\alpha_{s}$ between $\Lambda_{0}$ and $m$. A standard perturbative matching computation then leads to,

$$
m_{\phi}^{2} / \Lambda_{0}^{2} \sim\left(m / \Lambda_{0}\right)^{\frac{24}{29}} e^{-\frac{12 \pi}{29 \alpha_{s}\left(\Lambda_{0}\right)}}\left(\frac{m_{u}+m_{d}}{\Lambda_{0}}\right) .
$$

We see that $m_{\phi}$ is not unnaturally sensitive to $m$. Doubling $m$ only leads to a doubling of $m_{\phi}^{2}$, as compared to the extreme and unnatural sensitivity implied by the naive result, eq. (18). The quadratic sensitivity of scalar radiative corrections to the ultraviolet scale $m$ has been eliminated by having no scalar degree of freedom present at $m$, only quarks and gluons. These constituents of the scalar are only logarithmically sensitive to $m$.

To summarize, we were able to resolve the toy naturalness problem by giving the TG sector a very low compositeness scale and making the "gravitating" TSM particles into constituents of composite states. The toy composite "graviton" $\phi$ interacts with the compositeness "halo" that surrounds the TSM particles. At very low momenta this is indistinguishable from a direct coupling to the nearly on-shell TSM particles. If this is extrapolated to when the TSM particles are far off-shell, one runs into the naturalness problem. In reality though, the off-shell contributions are cut off by compositeness. The naive extrapolation misses this composite softness of the interactions in the TG dynamics. On the other hand the compositeness interactions are invisible in the lab, compared with the much stronger hard interactions of the TSM. The obvious regime to discover the compositeness dynamics is at intermediate distances, where TSM interactions are still neutralized but compositeness effects are unsuppressed in the TG dynamics. 


\section{The Effective Particle-String Scenario}

The moral of the previous section is that the power-counting that points to the inevitability of the CCP only holds if the graviton is fundamental, not if it is "composite". To exploit this observation we must ensure that there simply is no graviton at the energies at which we integrate out SM particles, en route to obtaining the long-distance theory of gravity. At these SM energies there should only be the degrees of freedom which will bind into the graviton at much lower energies. A second requirement is that the SM particles must couple to composite gravity, and yet their couplings to other SM particles must be point-like at least down to $1 / v$ distances. Unlike the case of the scalar $\phi$ in the toy model though, the compositeness of the graviton cannot be accomplished within the ordinary Minkowski space quantum field theory of point particles. This is due to the following very general theorem [14]: a theory in Minkowski space which admits a wellbehaved, conserved energy-momentum tensor cannot have a graviton in its spectrum.

Fortunately, string theory evades this theorem and gives a sensible meaning to graviton compositeness. Though formulated in Minkowski space, its energy-momentum tensor is not "well-behaved" and there is a massless graviton in the spectrum, as discussed in ref. 15. In terms of the wellknown similarity between string theory and QCD (which of course was important historically for the discovery of string theory [16]), the graviton can be thought of as a massless "glueball" of string theory. Now in QCD there are sum rules that can be derived in terms of the fundamental description which look miraculous or finely-tuned in terms of the hadronic description. They are not enforced by any symmetry but by the special nature of the dynamics. The same is true in string theory with respect to the cosmological constant.

For simplicity let us consider the case of the perturbative bosonic string in 26 dimensions [17]. There are effectively two parameters, the string massscale, $m_{s t}$, which plays the role of the graviton compositeness scale, and the string coupling, $g_{s t}$. For $g_{s t} \ll 1$, the string spectrum corresponds to an infinite number of "composite" particle-modes of varying spins and masses, including a graviton. The Planck scale is very large, $M_{P l} \gg m_{s t}$. If we ignore the string principle, we can compute the 1-loop contributions to the cosmological constant from each of the particles. Each contribution is quartically sensitive to the particle mass, and there are an infinite number of such contributions. Clearly we must introduce an ultraviolet cutoff, which 
cuts off both the infinity of contributions and the infinity in each contribution. This still leaves many large contributions to the cosmological constant. If we want the renormalized cosmological constant to come out very small, we must also add a counterterm chosen very precisely to finely cancel the large 1-loop contributions. Of course, in string theory the sum of one-loop diagrams plus counterterm is not calculated in this piecemeal fashion, but rather at one stroke. The result is an ultraviolet finite cosmological constant, $\lambda \sim m_{s t}^{26}$. Note, this is just the power-counting dependence on an ultraviolet cutoff, $m_{s t}$, in 26-dimensional general relativity. This illustrates our expectation that the compositeness scale should cut off the divergences of general relativity. Since the 26-dimensional Planck scale is given by

$$
M_{P l}=\frac{m_{s t}}{g_{s t}^{1 / 12}}
$$

$\lambda$ can be made arbitrarily small compared to $M_{P l}^{26}$ by taking small enough $g_{s t}$. In Planck units this corresponds to a very low tension string theory.

However, in the usual string formulation of particle physics, the SM particles are also identified as string vibrational modes, and we must have $m_{s t}>v$ so that the stringy excitations of the SM particles are too massive to appear in present-day experiments. Strings with such a large string-scale cannot solve the CCP. In fact we can integrate out the excited string states and return to eq. (5) and its unpleasant consequences. Instead, we wish to pursue the possibility that there are strings in the gravitational sector with extremely low string-scale, $m_{s t} \ll v$, but the SM particles are not themselves made of these strings. Instead SM particles are point-like, at least up to the weak scale. Just as the heavy quarks of the last section were surrounded by a light hadronic halo to form a heavy meson, the SM particles may be surrounded by a stringy halo with which the graviton string mode interacts.

As yet there are no fully realisitic candidates known within string theory, which are point-like on the string scale and can be identified with the SM particles. However the recently discovered solitonic D-branes [2] do possess some promising qualitative features. For example, 0-branes are point-like objects with masses much larger than $m_{s t}$, which can probe a continuum spacetime down to distances much shorter than $1 / m_{s t}$ [3]. At long distance their interactions conform to general relativistic expectations in terms of graviton exchange. At distances smaller than $1 / m_{s t}$ the composite graviton is an entirely inappropriate degree of freedom, and the force between 0branes becomes intrinisically stringy [3]. 
I therefore propose that in nature the SM particles are dynamically more akin to 0-branes than they are to perturbative string modes such as the graviton. Since string theories are only consistently formulated with supersymmetry, it remains a problem to explain how supersymmetry ends up badly broken in the SM sector. Nevertheless, supposing this is possible, we would like to explicitly understand how the cosmological constant can be cut off by the scale of graviton compositeness, $m_{s t}$, rather than being sensitive to the much larger SM masses. Below I offer a picture of how this might work. I can make no pretence of rigor.

\subsection{How the particle-string might solve the CCP}

Let us consider a simple, abstracted version of our problem. To eliminate the complication of supersymmetry-breaking and compactification, let us simply work within bosonic string theory in 26 euclidean dimensions (turning a blind eye to the existence of a tachyon). This will be our gravitational string sector. Let the 0 -brane of this theory represent a "SM particle", with mass $m \sim m_{s t} / g_{s t}$ [2]. For $g_{s t} \ll 1$, the 0 -branes are much more massive than the string scale. Strings are permitted to end on the 0-brane worldline, the attached string constituting a string "halo". Closed strings, including the graviton, are emitted and absorbed by this halo, inducing gravitational interactions for the 0-branes. We want to estimate the contributions of virtual 0-brane loops to the cosmological constant, $\lambda .10$ The naive powercounting guess, ignoring the string principle, would be $\lambda \sim m^{26}$. I will argue that the cosmological constant is instead set by the graviton compositeness scale, $m_{s t}$, so that $\lambda \sim m_{s t}^{26}$.

The simplifying consideration is that the euclidean action for a particle of mass, $m$, will suppress 0-brane world-line loops which are much bigger than $1 / m$. Thus on the string scale they are essentially point-like events in spacetime, to which string worldsheet boundaries can attach.円 In the string literature, such events are known as "D-instantons". The $1 / m_{\text {st }}$ sized strings should be insensitive to the tiny $1 / m$-scale structure.

The contribution to the cosmological constant due to D-instantons has

\footnotetext{
${ }^{10}$ Strictly speaking, the notion of a 0-brane perturbative loop expansion is ill-defined, since these 0-branes are so massive that their gravitational couplings are large. I will however use this language since it is the most familiar one, and because it is likely to apply to a more realistic construction.

${ }^{11}$ This is very much like ordinary quantum field theory, where integrating out a massive field introduces local interactions for the light fields.
} 
been computed and the result is finite and of order $e^{-1 / g_{s t}}$ [18]. This result can be understood as follows. The cosmological constant correction is given by the sum of (first-quantized) connected string diagrams with no vertex operators, where the string worldsheet boundaries attach to the Dinstanton. The dominant contribution from a single worldsheet is of order $1 / g_{s t}$, corresponding to a disk topology, more complicated topologies being suppressed by powers of $g_{s t}$. The sign of this contribution requires a detailed calculation and is negative. The dominant contribution from $k$ worldsheets is given by $k$ identical disks, whose boundaries attach to the D-instanton. Their contribution is just the $k$ th power of the single-disk result, divided by a symmetry factor of $k$ !. Summing over $k$ gives the factor $e^{-1 / g_{s t}}$.

Thus we expect that the contribution to the cosmological constant from 0 -brane loops is suppressed by $e^{-1 / g_{s t}}$ (without being careful about the prefactor) and is therefore negligible for $g_{s t} \ll 1$ ! Therefore the cosmological constant is dominated by the string-loop correction discussed earlier,

$$
\lambda \sim m_{s t}^{26} .
$$

\subsection{Phenomenological aspects of this scenario}

A fully realistic effective particle-string theory has not yet been constructed. I will just list some important features that it ought to have.

- The theory must contain SM particles and critical strings. The particles must live in four spacetime dimensions and be point-like at least down to $1 / v$ distances. The string length scale and compactification radii can however be much larger. Examples of four-dimensional particle-like behavior co-existing with strings, and large compactification radii "seen" only by the strings, have been found and discussed in refs. [19] [20].

- The theory must be unitary below the weak scale. It is permitted to break down above the weak scale, since we are not trying to guess the very high energy physics.

- The spin-2 graviton must be the only massless non-SM state with couplings to matter (unless they are even weaker than gravity). Then unitarity ensures that at long distances the dynamics reduces to general relativity [10] [16]. For distances of order $1 / m_{s t}$ or smaller, the massive string physics will become important and general relativity must break down. The fact that gravity has already been tested at distances of a few centimeters without deviation from Newton's Law, gives the bound,

$$
m_{s t}>10^{-5} \mathrm{eV} \text {. }
$$


- The compositeness of gravity must make the cosmological constant insensitive to the large SM masses, its size being set instead by the compositeness scale, $m_{s t}$,

$$
\lambda \sim \mathcal{O}\left(m_{s t}^{4}\right)
$$

This is also the power-counting result that follows from thinking of $m_{s t}$ as an ultraviolet cutoff for the effective theory of general relativity.

To satisfy the bound of eq. (4), we must have,

$$
m_{s t}<10^{-2} \mathrm{eV} \text {. }
$$

If the string compactification radii are of order $1 / m_{s t}$, the string coupling is given by,

$$
g_{s t}=m_{s t} / M_{P l}=10^{-30} .
$$

This may seem absurdly small, but recall that in string theory, $g_{s t}=$ $e^{-\langle\text {dilaton }\rangle}$, and the stabilization of the dilaton vev is still not understood. It may be related to the other absurdly small number in nature, $v / M_{P l} \sim$ $10^{-16}$. In any case, small $g_{s t}$ is not technically unnatural.

- The new stringy physics must be negligible in SM experiments. While the strings have typical length $1 / m_{s t}>10^{-1} \mathrm{~mm}$, their couplings are so incredibly weak that they should not interefere with the SM interactions. At lab momenta, the strings should form an insubstantial cloud about the SM particles. In particular, they should not upset the theoretical agreement with SM experiments which are sensitive to very small mass splittings, such as kaon mixing or atomic structure. This may be of concern given eq. (29).

- String theories are presently formulated with supersymmetry as an essential ingredient for full consistency, yet supersymmetry must appear broken by at least $v$ in the particle sector. This suggests a minimal supersymmetry breaking in the string sector of order $v^{2} / M_{P l}$. This scale may set the minimal permissible string-scale. If so, $m_{s t}>10^{-4} \mathrm{eV}$.

Finally, let us consider how this scenario might be experimentally tested. We expect that the nature of the gravitational force should dramatically change for distances smaller than the compositeness length scale, $1 / m_{s t}$, in a manner which cannot be described by the exchange of a finite variety of massive particles (such as the light scalars discussed in refs. [11] or 21], for example). For example, the interaction between a pair of 0-branes is described at long distance in terms of the exchange of massless closed string modes such as the graviton, while at short distance it is described by open 
strings connecting the 0-branes [2] [3]. In this regime the gravitational force can become weaker with shorter distances!

Eqs. (29) and (31) narrowly constrain the compositeness length scale at which the radical departures from general relativity (Newton's Law) must occur,

$$
10 \mu \mathrm{m}<1 / m_{s t}<1 \mathrm{~cm} .
$$

It is therefore our very good fortune that this is just the range over which gravity will be sensitively tested in the experiment proposed in ref. [22]. If composite gravity resolves the CCP as proposed here, it will show itself in this experiment and be quite distinct from any other "fifth force" phenomenon which can be described within field theory!

\section{Conclusions}

The Cosmological Constant Problem was argued to be intractable as a naturalness problem in effective field theory unless the graviton was "composite" with a scale of compositeness below $10^{-2} \mathrm{eV}$. The standard model particles must also participate in this compositeness and yet retain their point-like behavior in accelerator experiments up to very high energies. The only sensible version of graviton compositeness that is known, is string theory. It was proposed that the standard model particles inherit their gravitational interactions by virtue of their their stringy "halos", coupled to a gravitational string sector. The string-scale plays the role of the compositeness scale. It was argued that this stringiness can acceptably cut off contributions to the cosmological constant from ultraviolet mass scales. The mechanism is reminiscent of the relative insensitivity of light hadron masses to heavy quark masses in QCD. This was the basis for the detailed analogy discussed in the paper.

If this particle-string scenario is realized in nature, it will lead to a dramatic breakdown of Newton's Law on the millimeter scale, which will be experimentally probed. On the theoretical side, much work still remains in order to construct a fully realistic effective particle-string theory and demonstrate its requisite properties. The particle-string scenario considered here would obviously also have deep implications for physics at the highest energy scales.

Finally, it is worth keeping in mind that there may be other, presently undiscovered, manifestations of graviton compositeness that can also reduce 
the sensitivity of the cosmological constant to ultraviolet mass scales. Fortunately, independently of the form of graviton compositeness which resolves the CCP, power-counting suggests that eq. (33) constrains the compositeness length scale. Therefore, the composite behavior should still show up in upcoming experimental tests of gravity at short distances.

\section{Acknowledgments}

This research was supported by the U.S. Department of Energy under grant \#DE-FG02-94ER40818. I wish to thank Tom Banks, Sekhar Chivukula, Andrew Cohen, Nick Evans, Shamit Kachru, Martin Schmaltz and especially my father, R. M. Sundrum, and my wife, Jamuna Sundrum, for useful conversations on the subject of this paper.

\section{References}

[1] S. Weinberg, Rev. Mod. Phys. 61 (1989) 1.

[2] J. Polchinski, TASI Lectures on D-Branes, hep-th/9611050.

[3] M. R. Douglas, D. Kabat, P. Pouliot and S. H. Shenker, Nucl. Phys. B485 (1997) 85.

[4] A. Hashimoto and I. R. Klebanov, Nucl. Phys. Proc. Suppl. B55 (1997) 118.

[5] N. Ishibashi, Particle-Particle-String Vertex, hep-th/9609173; S. Hirano and Y. Kazama, Scattering of Closed String States from a Quantized D-Particle, hep-th/9612064; Y. Kazama, Scattering of Quantized Dirichlet Particles, hep-th/9705111.

[6] S. Weinberg, Gravitation and Cosmology, Wiley (1972).

[7] M. Veltman, in Methods of Field Theory, Les Houches (1975) 265.

[8] J. Donoghue, Phys. Rev. D50 (1994) 3874; Introduction to the Effective Field Theory description of Gravity, gr-qc/9512024.

[9] M. Wise, in CCAST Symp. on Particle Physics at the Fermi Scale (1993), hep-ph/9306277. 
[10] S. Weinberg, Phys. Rev. 135 (1964) B1049; Phys. Rev. 138 (1965) B988.

[11] S. Beane, Gen. Rel. Grav 29 (1997) 945.

[12] S. Coleman, Nucl. Phys. B310 (1988) 643.

[13] L. B. Okun, Nucl. Phys. B173 (1980) 1.

[14] S. Weinberg and E. Witten, Phys. Lett. B96 (1980) 59.

[15] M. Karliner, I. Klebanov and L. Susskind, Int. J. Mod. Phys. 3 (1988) 1981; I. Klebanov and L. Susskind, Nucl. Phys. B309 (1988) 175.

[16] M. B. Green, J. H. Schwarz and E. Witten, Superstring Theory, Cambridge University Press (1987) chapter 1.

[17] J. Polchinski, Commun. Math. Phys. 104 (1986) 37. Academic Publishers, London (1987).

[18] J. Polchinski, Phys. Rev. D50 (1994) 6041.

[19] A. Strominger, Nucl. Phys. B451 (1995) 96.

[20] S. Shenker, Another Length Scale in String Theory, hep-th/9509132.

[21] S. Dimopoulos and G. F. Giudice, Phys. Lett. B379 (1996) 105.

[22] J. C. Price, in Proc. Int. Symp. on Experimental Gravitational Physics, Guangzhou, China (1988). 